\title{
Homotopy Analysis Investigation of Heat and Mass Transfer Flow Past a Vertical Porous Medium in the Presence of Heat Source
}

\author{
Bayo J. Akinbo*, Bakai I. Olajuwon \\ Department of Mathematics, Federal University of Agriculture, Abeokuta 110252, Nigeria
}

Corresponding Author Email: akinbomaths@gmail.com

https://doi.org/10.18280/ijht.370328

Received: 12 June 2019

Accepted: 3 September 2019

\section{Keywords:}

heat source, magnetic field, heat and mass transfer, homotopy analysis method, similarity solution

\begin{abstract}
In this paper, homotopy analysis method with two auxiliary parameters is used to investigate heat and mass transfer flow past a vertical porous medium in the presence of heat source. Similarity transformation is adopted to transform the boundary-layer coupled non-linear partial differential equation to a system of coupled non-linear ordinary differential equation. The resulting equations are then solved analytically. The effect of various flow parameters on velocity, temperature and concentration profiles are presented graphically and discussed while the local skin-friction, Nusselt number, plate surface temperature and Sherwood number are illustrated numerically in tabular form. The results show among all other obtained that the cooling problem is guaranteed with the positive values of Grashof number while the higher values of heat source energies the random movement of the fluid molecules and pave way for the penetration of the thermal effect to the quiescent fluid.
\end{abstract}

\section{INTRODUCTION}

Analysis of magnetohydrodynamic (MHD) flow with the coupled heat and mass transfer over a vertical plate with embedded porous medium, in the presence of heat source has great importance due to the influence of magnetic field in the boundary layer flow such as geothermal energy extraction and MHD generator etc. In Lieus of its numerous applications in science and engineering, it has been studied in the literature

Thommaandru et al. [1] studied effect of heat source/ sink on heat and mass transfer of magneto-nanofluids over a nonlinear stretching sheet. Ibrahim et al [2] investigated the effect of chemical reaction and radiation absorption on unsteady MHD mixed convection flow past a semi infinite vertical permeable moving plate with heat source and suction. Bakr [3] reported the effect of chemical reaction on MHD free convection and mass transfer flow of a micropolar fluid with oscillatory plate velocity and constant heat source in a rotating frame of reference. The internal heat generation effect on thermal boundary layer with a convective surface boundary condition was investigated by Olarewaju et al. [4]. Crepeau and Clarksean [5] worked on similarity solutions of natural convection with internal heat generation. Ashwini and Eswara [6] studied MHD Falkner Skan boundary layer flow with internal heat generation or absorption. Makinde [7] reported the similarity solution for natural convection from a moving vertical plate with internal heat generation and a convective boundary condition. By virtual of the endless impotances of heat generation or heat source with different conditions imposed on it, other authors like Azim et al. [8], Rashid and Waqar [9], Machireddy [10], Rena and Bhargav [11] and Kashmani et al. [12] considered the effect of heat source/ heat generation in their investigation. Considering the effect or impact of heat source or heat generation on MHD flow, the porosity part has not been left out due to its vast applications in science and engineering disciplines. Good numbers of work have been considered with the porosity parameter in the literature by different authors among which are; Mohammed et al. [13] investigated the thermal and MHD effect on free convective flow of a polar fluid through a porous medium in the presence of internal heat generation and chemical reaction. Patil and Kulkarini [14] worked on effect of chemical reaction on free convective flow of a polar fluid through a porous medium in the presence of internal heat generation. Ramana et al. [15] investigated mass transfer and radiation effects of unsteady MHD free convection fluid flow embedded in porous medium with heat generation/ absorption. Sharm and singh [16] discussed unsteady MHD free convective flow and heat transfer along a vertical porous with variable suction and internal heat generation. Mamta and Krishna [17] discussed the thermal radiation effect on an unsteady MHD free convection chemically reacting viscous dissipation fluid flow past an infinite vertical moving porous plate with heat source. Other authors like Sharma et al. [18] considered heat generation or heat source in their investigation.

The main purpose of this present paper is to investigate heat and mass transfer in hydromagnetic flow past a vertical porous media in the presence of heat source via homotopy analysis method discovered by Liao [19]. The effects of different involved parameters are discussed numerically and graphically.

\section{MATHEMATICAL FORMULATION}

We consider a steady-state heat and mass transfer flow of a stream of cold fluid at temperature $T_{\infty}$ that takes place in the presence of heat source. The left surface is considered to be heated by convection from the hot fluid at temperature $T_{f}$ that 
brings about heat transfer coefficient $h_{f}$. The cold fluid in contact with the surface of the plate produces heat internally at volumetric rate $Q_{0}$. The plate is subjected to a magnetic field of strength $B_{0}$ which act in a transverse direction to the flow and the effect of induced magnetic field is neglected. The $x$-axis is taken parallel to the plate direction and $y-$ axis normal to it. The fluid temperature and concentration are respectively taken as $\mathrm{T}$ and $\mathrm{C}$, while $C_{w}$ is the species concentration at the surface of the plate.

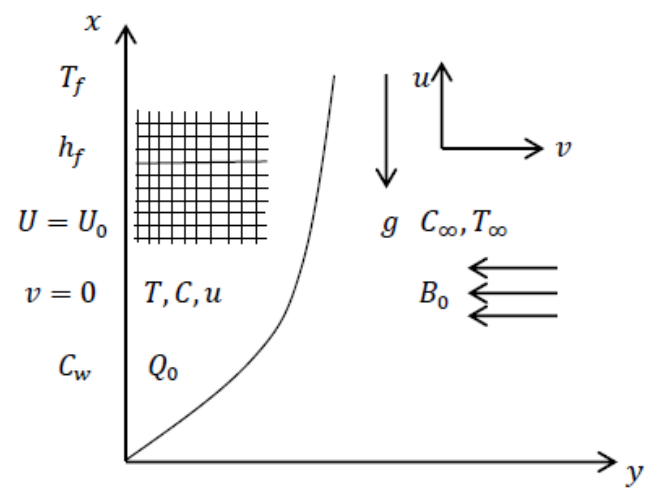

Figure 1. Flow configuration and coordinate system

In line with the assumption stated above and usual Boussinesq's approximation, the steady flow is governed by the equation slated below

$$
\begin{gathered}
\frac{\partial u}{\partial x}+\frac{\partial v}{\partial y}=0 \\
u \frac{\partial u}{\partial x}+v \frac{\partial u}{\partial y}=v \frac{\partial^{2} u}{\partial y^{2}}-\frac{\sigma B_{0} u}{\rho}-\frac{v}{K} u+g \beta_{T}\left(T-T_{\infty}\right)+ \\
g \beta_{c}\left(C-C_{\infty}\right) \\
u \frac{\partial T}{\partial x}+v \frac{\partial T}{\partial y}=\alpha \frac{\partial^{2} T}{\partial y^{2}}+\frac{Q_{0}\left(T-T_{\infty}\right)}{\rho C_{p}} \\
u \frac{\partial C}{\partial x}+v \frac{\partial C}{\partial y}=D \frac{\partial^{2} C}{\partial y^{2}}
\end{gathered}
$$

where, $(u, v)$ are components of the velocity at any point $(x, y), \mathrm{T}$ is the temperature of the fluid, $C$ is the concentration, $U_{0}$ is the plate velocity and $D, \alpha, T_{\infty}, C_{\infty}, g, \rho$, $\sigma, \beta_{T}, Q_{0}, C_{p}, \mathrm{v}, \beta_{c}$ and $B_{0}$ are the mass diffusivity, thermal diffusivity, free stream temperature, free stream concentration, acceleration due to gravity, density, fluid electrical conductivity, thermal expansion coefficient, volumemetric heat generation/absorption rate, specific heat at constant pressure, kinematics viscosity, concentration expansion coefficient and magnetic field of strength respectively. The appropriate boundary conditions at the surface of the plate and away into the cold fluid are expressed as follows

$$
\begin{gathered}
U(x, 0)=U_{0}, V(x, 0)=0,-k \frac{\partial T(x, 0)}{\partial y}=h_{f}\left[T_{f}-T(x, 0)\right] \\
C_{w}(x, 0)=A x^{\lambda}+C_{\infty}, U(x, \infty)=0 \\
T(x, \infty)=T_{\infty}, C(x, \infty)=C_{\infty}
\end{gathered}
$$

where, $k$ represents the thermal conductivity coefficient and $\lambda$ denotes the power index of the concentration. The continuity
Eq. (1) is satisfied automatically by invoking the stream function defined by

$$
u=\frac{\partial \psi}{\partial y} \quad \text { and } \quad v=-\frac{\partial \psi}{\partial x}
$$

Considering $\eta$ as independent variable and $f$ as dependent variable interms of stream function, similarity solution of (1$6)$ is given as

$$
\eta=y \sqrt{\frac{U_{0}}{v x}}, \quad \psi=\sqrt{v x U_{0}} f(\eta)
$$

where, $U_{0}$ represents the velocity of the plate and

$$
\theta(\eta)=\frac{T-T_{\infty}}{T_{f}-T_{\infty}}, \quad \emptyset(\eta)=\frac{C-C_{\infty}}{C_{w}-C_{\infty}}
$$

respectively denotes the dimensionless temperature and concentration. Applying Eqns. (6-8) into Eqns. (1-5) with the following quantities

$$
\begin{gathered}
H a=\frac{\sigma B_{0}^{2} x}{\rho U_{0}}, G r=\frac{g \beta_{T}\left(T_{f}-T_{\infty}\right) x}{U_{0}^{2}}, G c=\frac{g \beta_{c}\left(C_{w}-C_{\infty}\right) x}{U_{0}^{2}}, \\
B i=\frac{h f}{k} \sqrt{\frac{v x}{U_{0}}}, P r=\frac{v \rho C_{\rho}}{K^{*}}, \quad S c=\frac{v}{D} \\
\delta=\frac{x Q_{0} \mathrm{v}}{K^{*} U_{0}}, \quad P_{s}=\frac{\mathrm{v} x}{K U_{0}}, \alpha=\frac{K^{*}}{\rho C_{\rho}}
\end{gathered}
$$

we have

$$
\begin{gathered}
\frac{d^{3} f}{d \eta^{3}}+\frac{1}{2} f \frac{d^{2} f}{d \eta^{2}}-\left(H a+P_{S}\right) \frac{d f}{d \eta}+G r \theta(\eta)+G c \emptyset(\eta)=0 \\
\frac{d^{2} \theta}{d \eta^{2}}+\frac{1}{2} \operatorname{Pr} f \frac{d \theta}{d \eta}+\delta \theta(\eta)=0 \\
\frac{d^{2} \emptyset}{d \eta^{2}}+\frac{1}{2} S c f \frac{d \emptyset}{\partial \eta}=0
\end{gathered}
$$

Here, $\mathrm{Ha}$ is the local magnetic field parameter, $\mathrm{Gr}$ is the local thermal Grashof number, $G c$ is the Solutal Grashof number, $B i$ is the local convective heat transfer parameter, $P r$ is the Prandtl number, $S c$ is the Schmidt number, $\delta$ is the heat source and $P_{S}$ is the Porosity parameter. The corresponding boundary conditions are as follows

$$
\begin{gathered}
f(0)=0, f^{\prime}(0)=1, \theta^{\prime}(0)=B i[\theta(0)-1], \emptyset(0)=1 \\
f^{\prime}(\infty)=0, \quad \theta(\infty)=0, \quad \emptyset(\infty)=0
\end{gathered}
$$

The local parameters $H a, B i, G r, G c, \delta$ and $P_{s}$ in (10-12) denotes the function of $x$. In an attempt to have similarity solution, we assume the following parameters

$$
\sigma=\frac{b}{x}, h_{f}=\frac{a}{\sqrt{x}}, \beta_{T}=\frac{c}{x}, \beta_{c}=\frac{d}{x}, Q_{0}=\frac{e}{x}, K=\frac{x}{q}
$$

where, $a, b, c, d, e$ and $q$ are constant under the appropriate dimension. The nonlinear Eqns. (10-12) subject to the boundary conditions of Eqns. (13) and (14) are solved analytically by Homotopy Analysis Method as shown in (3.0) below. The rate of the model, corresponding to the local skin- 
friction, rate of heat transfer corresponding to the Nusselt number and rate of mass transfer corresponding to the Sherwood number are respectively considered, given by

$$
C_{f}=\frac{2 \tau_{w}}{\rho U_{0}^{2}}, \quad N u=\frac{x q_{w}}{k\left(T_{w}-T_{\infty}\right)}, \quad S h=\frac{x q_{m}}{D\left(C_{w}-C_{\infty}\right)}
$$

and expressed

$C_{f}=2 R e_{x}^{\frac{-1}{2}} f^{\prime \prime}(0), N u=-R e_{x}^{\frac{1}{2}} \theta^{\prime}(0), S h=-R e_{x}^{\frac{1}{2}} \emptyset^{\prime}(0)$

where, $R e_{x}=U_{0} x / v$ is the Reynold number, $\tau_{w}$ is the shear stress along the plate, $q_{w}$ is the surface heat and $q_{m}$ is the surface mass. Their numerical computation, in addition with the plate plate surface temperature which is denoted by $\theta(0)$ are presented in the Table 2 .

\section{HOMOTOPY ANALYSIS METHOD}

Homotopy Analysis Method (HAM), discovered by Liao [19-20] is preferred over another method and adopted for this work due to its efficiency in solving both Linear and nonlinear differential equations. The non-linear differential equations are usually inevitable and have become a culture in our daily mathematical modeling. They are solved by different methods, among which are; differential transform method (DTM), Variation Iteration Method and so on. Following Olubode et al. [21] in accordance with the rule of solution and boundary conditions (13)-(14), we choose the initial guess

$$
\begin{gathered}
f_{0}(\eta)=1-\exp (-\eta), \quad \theta_{0}(\eta)=\frac{B i \exp (-\eta)}{(1+B i)} \\
\emptyset_{0}(\eta)=\exp (-\eta)
\end{gathered}
$$

as the initial linear approximations of $f(\eta), \theta(\eta)$ and $\emptyset(\eta)$ and the auxiliary linear operations $L_{f}, L_{\theta}$, and $L_{\varnothing}$ as;

$$
\begin{aligned}
& L_{f}[f(\eta ; r)]=\frac{\partial^{3} f(\eta ; r)}{\partial \eta^{3}}-\frac{\partial f(\eta ; r)}{\partial \eta} \\
& L_{\theta}[\theta(\eta ; r)]=\frac{\partial^{2} \theta(\eta ; r)}{\partial \eta^{2}}-\theta(\eta ; r) \\
& L_{\emptyset}[(\eta ; r)]=\frac{\partial^{2} \emptyset(\eta ; r)}{\partial \eta^{2}}-\emptyset(\eta ; r)
\end{aligned}
$$

agreed with the following properties

$$
\begin{gathered}
L_{f}\left[C_{1}+C_{2} \exp (\eta)+C_{3} \exp (-\eta)\right]=0 \\
L_{\theta}\left[C_{4}+C_{5} \exp (-\eta)\right]=0 \\
L_{\varnothing}\left[C_{6}+C_{7} \exp (-\eta)\right]=0
\end{gathered}
$$

where, $C_{1}, C_{2}, \ldots, C_{7}$ are constants.

\subsection{Zero order deformation problem}

$$
\begin{gathered}
(1-r) L_{f}\left[f(\eta ; r)-f_{0}(\eta)\right] \\
=r \hbar_{f} H_{f}(\eta) N_{f}[f(\eta ; r), \theta(\eta ; r), \emptyset(\eta ; r)] \\
(1-r) L_{\theta}\left[f(\eta ; r)-\theta_{0}(\eta)\right]
\end{gathered}
$$

$$
\begin{aligned}
= & r \hbar_{\theta} H_{\theta}(\eta) N_{\theta}[f(\eta ; r), \theta(\eta ; r)] \\
& (1-r) L_{\varnothing}\left[f(\eta ; r)-\emptyset_{0}(\eta)\right] \\
= & r \hbar_{\varnothing} H_{\varnothing}(\eta) N_{\varnothing}[f(\eta ; r), \emptyset(\eta ; r)]
\end{aligned}
$$

having the following boundary conditions.

$$
\begin{gathered}
f(\eta=0 ; r)=0,\left.\frac{\partial f(\eta ; r)}{\partial \eta}\right|_{\eta=0}=1, \\
\left.\frac{\partial \theta(\eta ; r)}{\partial \eta}\right|_{\eta=0}=B i[\theta(\eta=0 ; r)-1], \emptyset(\eta=0 ; r)=1 \\
\left.\frac{\partial f(\eta ; r)}{\partial \eta}\right|_{\eta \rightarrow \infty}=0, \quad \theta(\eta \rightarrow \infty ; r)=0=\emptyset(\eta \rightarrow \infty ; r)
\end{gathered}
$$

where, $N_{f}, N_{\theta}$, and $N_{\varnothing}$ are nonlinear operator defined as

$$
\begin{gathered}
N_{f}[f(\eta ; r), \theta(\eta ; r), \emptyset(\eta ; r)] \\
=\frac{\partial^{3} f(\eta ; r)}{\partial \eta^{3}}+\frac{1}{2} f(\eta ; r) \frac{\partial^{2} f(\eta ; r)}{\partial \eta^{2}}-\left(H a+P_{s}\right) \frac{\partial f(\eta ; r)}{\partial \eta} \\
G r \theta(\eta ; r)+G c \emptyset(\eta ; r) \\
N_{\theta}[f(\eta ; r), \theta(\eta ; r)] \\
=\frac{\partial^{2} \theta(\eta ; r)}{\partial \eta^{2}}+\frac{1}{2} \operatorname{Pr} \frac{\partial \theta(\eta ; r)}{\partial \eta} f(\eta ; r)+\delta \theta(\eta ; r)
\end{gathered}
$$

where, $r \in[0,1]$ is the embedding parameter and $\hbar_{f} \neq 0, \hbar_{\theta} \neq$ 0 and $\hbar_{\emptyset} \neq 0$ are the auxiliary parameter

\subsection{Mth-order deformation problem}

The increase in embedding parameter $r$ from Zero to One, lead to a variation of the function $f(\eta ; r), \theta(\eta ; r)$ and $\emptyset(\eta ; r)$ from initial guess $f_{0}(\eta), \theta_{0}(\eta)$ and $\emptyset_{0}(\eta)$ to the solutions $f(\eta ; r), \theta(\eta ; r)$ and $\varnothing(\eta ; r)$. Using Taylor series with respect to $r$, we have

$$
\begin{aligned}
& f(\eta ; r)=f_{0}(\eta)+\sum_{m=1}^{\infty} f_{m}(\eta) r^{m} \\
& \theta(\eta ; r)=\theta_{0}(\eta)+\sum_{m=1}^{\infty} \theta_{m}(\eta) r^{m} \\
& \emptyset(\eta ; r)=\emptyset_{0}(\eta)+\sum_{m=1}^{\infty} \emptyset_{m}(\eta) r^{m}
\end{aligned}
$$

where,

$$
\begin{gathered}
f_{m}(\eta)=\frac{1}{m !} \frac{\partial^{m} f(\eta ; r)}{\partial \eta^{m}}, f_{m}(\eta)=\frac{1}{m !} \frac{\partial^{m} \theta(\eta ; r)}{\partial \theta^{m}}, \\
f_{m}(\eta)=\frac{1}{m !} \frac{\partial^{m} \emptyset(\eta ; r)}{\partial \emptyset^{m}},
\end{gathered}
$$

Obviously, the convergences of the series (33)-(35) are subject to the auxiliary parameter $\hbar$. Assuming $\hbar$ is chosen such that the series (33)-(35) converge at $r=1$, we have

$$
\begin{gathered}
f(\eta)=f_{0}(\eta)+\sum_{m=1}^{\infty} f_{m}(\eta), \theta(\eta)=\theta_{0}(\eta)+\sum_{m=1}^{\infty} \theta_{m}(\eta), \\
\emptyset(\eta)=\emptyset_{0}(\eta)+\sum_{m=1}^{\infty} \emptyset_{m}(\eta)
\end{gathered}
$$


For the mth-order deformation, we take the derivative of zeroth-order deformation of Eqns. (25)-(27) times with respect to $r$, dividing by $m$ ! and set $r=0$, we have

$$
\begin{gathered}
L_{f}\left[f_{m}(\eta)-\chi_{m} f_{m-1}(\eta)\right]=\hbar R_{m}^{f}(\eta) \\
L_{\theta}\left[\theta_{m}(\eta)-\chi_{m} \theta_{m-1}(\eta)\right]=\hbar R_{m}^{\theta}(\eta) \\
L_{\varnothing}\left[\emptyset_{m}(\eta)-\chi_{m} \emptyset_{m-1}(\eta)\right]=\hbar R_{m}^{\emptyset}(\eta)
\end{gathered}
$$

having the following boundary conditions.

$$
\begin{gathered}
f_{m}(\eta=0 ; 0)=0, \quad \frac{\partial f_{m}(\eta=0 ; 0)}{\partial \eta}=0, \\
\frac{\partial \theta_{m}(\eta=0 ; 0)}{\partial \eta}=B i\left[\theta_{m}(\eta=0 ; 0)\right], \\
\emptyset_{m}(\eta=0 ; 0)=0 \\
\frac{\partial f_{m}(\eta \rightarrow \infty)}{\partial \eta}=0, \theta_{m}(\eta \rightarrow \infty)=0=\emptyset_{m}(\eta \rightarrow \infty)
\end{gathered}
$$

where,

$$
\begin{array}{r}
R_{m}^{f}(\eta)=\frac{d^{3} f_{m-1}(\eta)}{d \eta^{3}}+\frac{1}{2} \sum_{n=0}^{m-1} f_{n}(\eta) \frac{d^{2} f_{m-1-n}(\eta)}{d \eta^{2}} \\
-\left(H a+P_{s}\right) \frac{d f_{m-1}(\eta)}{d \eta} G r \theta_{m-1}+G c \emptyset_{m-1} \\
R_{m}^{\theta}(\eta)=\frac{d^{2} \theta_{m-1}(\eta)}{d \eta^{2}}+\frac{1}{2} \operatorname{Pr} \sum_{n=0}^{m-1} f_{n}(\eta) \frac{d \theta_{m-1-n}(\eta)}{d \eta} \\
+\delta \theta_{m-1} \\
R_{m}^{\emptyset}(\eta)=\frac{d^{2} \emptyset_{m-1}(\eta)}{d \eta^{2}}+\frac{1}{2} S c \sum_{n=0}^{m-1} f_{n}(\eta) \frac{d \emptyset_{m-1-n}(\eta)}{d \eta}
\end{array}
$$

and $\chi_{m}=0 \quad$ for $m \leq 1 ; \quad \chi_{m}=1$ for $m>1$

having the following as a general solution

$$
\begin{gathered}
f_{m}(\eta)=f_{m}^{*}(\eta)+C_{1}+C_{2} \exp (-\eta)+C_{3} \exp (\eta) \\
\theta_{m}(\eta)=\theta_{m}^{*}(\eta)+C_{4}+C_{5} \exp (\eta) \\
\emptyset_{m}(\eta)=\emptyset_{m}^{*}(\eta)+C_{6}+C_{7} \exp (\eta)
\end{gathered}
$$

where, $f_{m}^{*}(\eta), \theta_{m}^{*}(\eta)$ and $\emptyset_{m}^{*}(\eta)$ represent the particular solution of equations (40) and (41). In agreement with Adhikari [22], we consider the rule of coefficient ergodicity and rule of solution existence and choose the auxiliary functions as

$$
H_{f}=H_{\theta}=H_{\emptyset}=1
$$

\subsection{Convergence of the HAM solution}

The convergence of this present investigation was considered in-line with Liao [19]. The series equations (33)(35) which consist of non-zero auxiliary $\hbar_{f}, \hbar_{\theta}$ and $\hbar_{\varnothing}$ play important roles in adjusting and control the convergence. However, the admissible range values for $\hbar_{f}, \hbar_{\theta}$ and $\hbar_{\varnothing}$, are obtained at the 10th-order approximation of HAM with $H a=0.1, G r=0.1, G c=0.1, P r=0.72, S c=0.62, B i=$
$0.1, \delta=0.05, P_{s}=0.1$ at the range where $\hbar$-curve becomes parallel which gives $-1.8 \leq \hbar_{f} \leq-0.4,-2.2 \leq$ $\hbar_{\theta} \leq-0.2$, and $-1.8 \leq \hbar_{\emptyset} \leq-0.5$ which respectively justify the convergence of $f^{\prime \prime}(0), \theta^{\prime}(0)$ and $\emptyset^{\prime}(0)$ as shown in Figures (2-4)

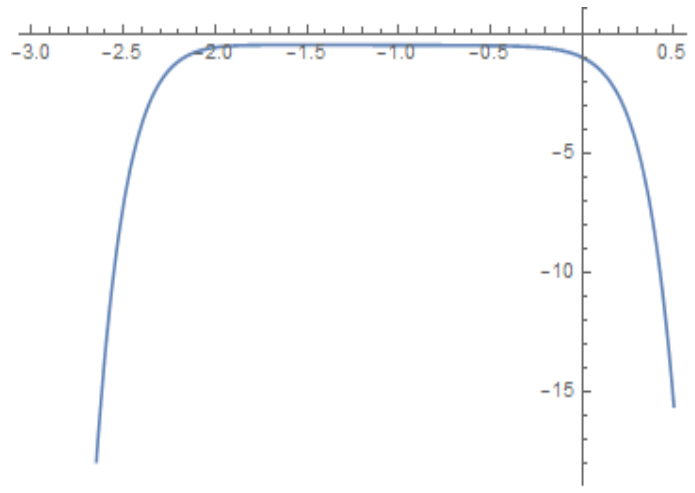

Figure 2. $\hbar_{f}$-curve of $f^{\prime \prime}(0)$ at 10 th order of approximation

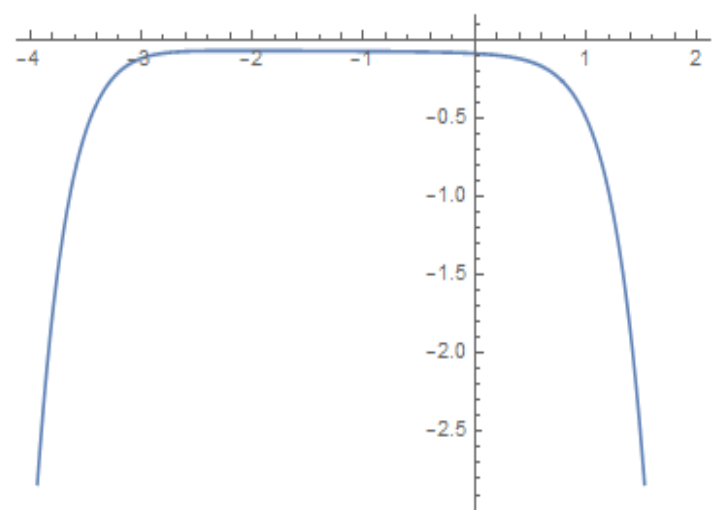

Figure 3. $\hbar_{\theta}$-curve of $\theta^{\prime}(0)$ at 10th order of approximation

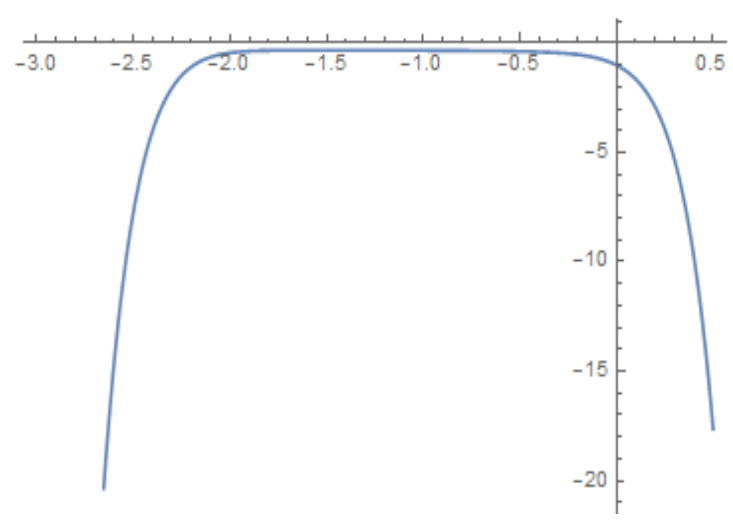

Figure 4. $\hbar_{f}$-curve of $\emptyset^{\prime}(0)$ at 10 th order of approximation

\section{VALIDATION OF THE STUDY}

The successful implementation of the numerical results was first considered by comparing it with the previous work done in the literature. So, these present results are compared to those obtained by Makinde [23] for the local skin-friction, Nusselt Number, plate surface temperature and Sherwood number by setting $\boldsymbol{P}_{\boldsymbol{s}}=\mathbf{0}$ and $\boldsymbol{\delta}=\mathbf{0}$ The results strongly agreed with each other (see Table 1). This shows that there is no deficiency with the method. 
Table 1. Comparison of the present result with Makinde [23]

\begin{tabular}{|c|c|c|c|c|c|c|c|c|c|c|c|c|c|}
\hline & & & & & & \multicolumn{4}{|c|}{ Makinde [23] } & \multicolumn{4}{|c|}{ Present result } \\
\hline $\mathrm{Ha}$ & $G r$ & $G c$ & $B i$ & $P r$ & $S c$ & $f^{\prime \prime}(0)$ & $-\theta^{\prime}(0)$ & $\theta(0)$ & $-\emptyset^{\prime}(0)$ & $f^{\prime \prime}(0)$ & $-\theta^{\prime}(0)$ & $\theta(0)$ & $-\phi^{\prime}(0)$ \\
\hline 0.1 & 0.1 & 0.1 & 0.1 & 0.72 & 0.62 & -0.402271 & 0.078635 & 0.213643 & 0.3337425 & -0.402272 & 0.078636 & 0.213644 & 0.3337425 \\
\hline 1.0 & 0.1 & 0.1 & 0.1 & 0.72 & 0.62 & -0.352136 & & & & -0.352137 & & & \\
\hline 10 & & 0. & 0.1 & 0.72 & 0.62 & -0.32 & & & & -0.32 & & & \\
\hline 0.1 & 0.5 & 0.1 & 0.1 & 0.72 & 0.62 & -0.32 & & & & -0.322 & & & \\
\hline 0.1 & 1.0 & 0.1 & 0.1 & 0.72 & 0.62 & -0.231 & & & & & & & \\
\hline 0.1 & 0.1 & 0.5 & 0.1 & 0.72 & 0.62 & -0.0264 & 0.080 & 0.19 & & -0.026 & 0.080 & 889 & \\
\hline 0.1 & 0.1 & 1.0 & 0.1 & 0.72 & 0.62 & 0.3799184 & 0.082040 & 0.179 & 6697 & 0.3799185 & 0.082 & 593 & \\
\hline 0.1 & 0.1 & 0.1 & 1.0 & 0.72 & 0.62 & -0.985719 & & & & -0.985 & & & 0.2598500 \\
\hline 0.1 & 0.1 & 0.1 & 5.0 & 0.72 & 0.62 & -2.217928 & 0.066156 & 0.338 & & -2.217 & 0.06 & & 0.1806634 \\
\hline 0.1 & 0.1 & 0.1 & 0.1 & 1.00 & 0.62 & -0.407908 & & & & -0.407909 & & & 0.3325180 \\
\hline 0.1 & 0.1 & 0.1 & 0.1 & 7.10 & 0.62 & -0.421228 & 0.093348 & 0.066513 & & -0.421229 & 0.093349 & 0.066514 & 0.3305619 \\
\hline 0.1 & 0.1 & 0.1 & 0.1 & 0.72 & 0.78 & -0.411704 & 0.078484 & 0.215159 & 0.3844559 & -0.411705 & 0.078485 & 0.215160 & 0.3844560 \\
\hline
\end{tabular}

\section{DISCUSSION OF RESULTS}

In the light of physical understanding of this present problem, the set of coupled nonlinear Eqns. (10)-(12) governing the problem with the boundary conditions (13) and (14) have been solved by Homotopy Analysis Method (HAM) at 20th-order because of the unbounded domain, in order to meet the far field boundary conditions.

The resulting effects of various parameters in the flow system such as: Magnetic Parameter $(\mathrm{Ha})$, Thermal Grashof Number $(G r)$, Solutal Grashof Number $(G c)$, Prandtl Number $(P r)$, Schmidt Number $(S c)$, Local Heat transfer parameter $(B i)$, Heat Source Parameter $(\delta)$ and Porosity parameter $\left(P_{s}\right)$ on Velocity profile, Temperature profile, Concentration profile and Local Skin-friction, Local Nusselt Number, plate surface temperature, Sherwood number were presented graphical and tabular form.

The values of Schmidt number $S c$ for diffusing chemical species in air were chosen to be $S c=0.24\left(\mathrm{H}_{2}\right)$, $0.62\left(\mathrm{H}_{2} \mathrm{O}\right), \mathrm{Sc}=0.78\left(\mathrm{NH}_{3}\right)$ and $\mathrm{Sc}=2.62\left(\mathrm{C}_{9} \mathrm{H}_{12}\right)$.

The Prandtl number was taken to be 0.72 which correspond to air and it is mostly encountered fluid in nature and commonly used in engineering. For the purpose of numerical calculation, other parameters were chosen to be $H a=0.1, G r=0.1, G c=0.1, P_{s}=0.1, B i=0.1, \delta=$ 0.05 .

The effect of magnetic parameter on the velocity, temperature and concentration profiles are shown in Figure 5-7. Obviously from Figure 5, velocity distribution across the boundary layer decreases as magnetic parameter increases. This quantitatively agrees with the expectation because the presence of $\mathrm{Ha}$ in an electrically conducting fluid produces a drag-like force called Lorentz force which act against the flow, thereby resist the motion of fluid flow and enhances the heat energy of which its aftermath effect increases the fluid temperature and its concentration. This inturns enhances the thermal and concentration boundary layers.

The enhancement in fluid concentration which in turns increases its boundary layer thickness is justified by the temperature gradient inherent in the viscosity of the fluid. It can be seen from Table 2 that the magnitude of the local skin-friction improves due to an increase in magnetic interaction. This in turns improves the shear stress and enhance flow.

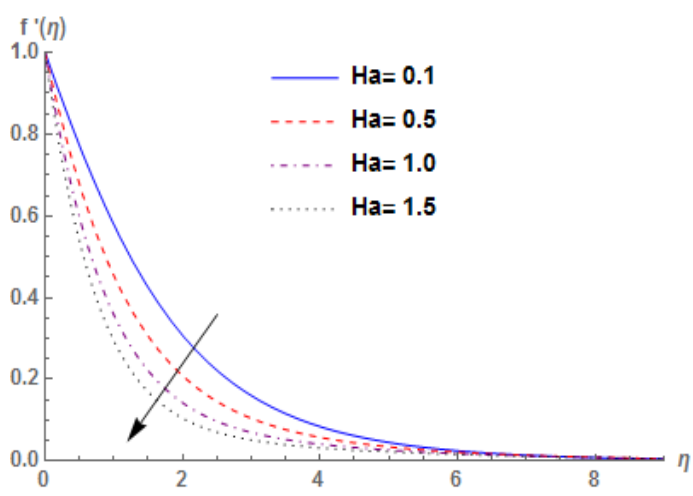

Figure 5. Velocity profiles for different values of $\mathrm{Ha}$

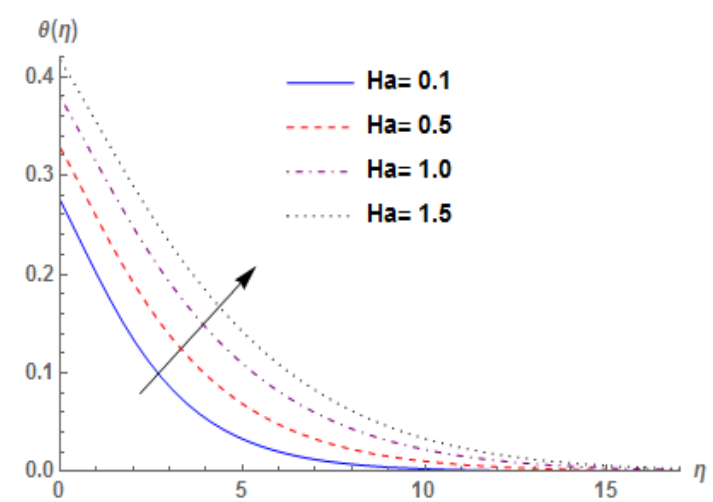

Figure 6. Temperature profiles for different values of $\mathrm{Ha}$

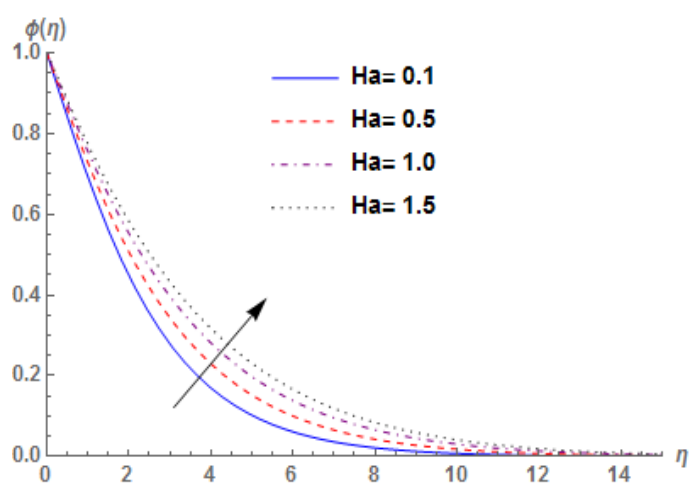

Figure 7. Concentration profiles for different values of $\mathrm{Ha}$ 
Table 2. Numerical values of the Skin-friction coefficient, local Nusselt number, local Sherwood number and plate surface temperature

\begin{tabular}{|c|c|c|c|c|c|c|c|c|c|c|c|}
\hline $\mathrm{Ha}$ & $G r$ & $G c$ & $B i$ & $\delta$ & $P r$ & $S c$ & $P s$ & $f^{\prime \prime}(0)$ & $-\theta^{\prime}(0)$ & $\theta(0)$ & $-\emptyset^{\prime}(0)$ \\
\hline 0.1 & 0.1 & 0.1 & 0.1 & 0.05 & 0.72 & 0.62 & 0.1 & 0.480589 & 0.072495 & 0.275054 & 0.321378 \\
\hline 0.5 & 0.1 & 0.1 & 0.1 & 0.05 & 0.72 & 0.62 & 0.1 & 0.759608 & 0.067168 & 0.328319 & 0.283825 \\
\hline 1.0 & 0.1 & 0.1 & 0.1 & 0.05 & 0.72 & 0.62 & 0.1 & 1.022340 & 0.062071 & 0.379290 & 0.254589 \\
\hline 0.1 & 0.1 & 0.1 & 0.1 & 0.05 & 0.72 & 0.62 & 0.1 & 0.480589 & 0.072495 & 0.275054 & 0.321378 \\
\hline 0.1 & 0.5 & 0.1 & 0.1 & 0.05 & 0.72 & 0.62 & 0.1 & 0.380176 & 0.074430 & 0.255701 & 0.338241 \\
\hline 0.1 & 1.0 & 0.1 & 0.1 & 0.05 & 0.72 & 0.62 & 0.1 & 0.272045 & 0.075709 & 0.242913 & 0.352242 \\
\hline 0.1 & 0.1 & 0.1 & 0.1 & 0.05 & 0.72 & 0.62 & 0.1 & 0.480589 & 0.072495 & 0.275054 & 0.321378 \\
\hline 0.1 & 0.1 & 0.5 & 0.1 & 0.05 & 0.72 & 0.62 & 0.1 & 0.120729 & 0.077239 & 0.227614 & 0.370425 \\
\hline 0.1 & 0.1 & 1.0 & 0.1 & 0.05 & 0.72 & 0.62 & 0.1 & 0.273632 & 0.079484 & 0.205156 & 0.407670 \\
\hline 0.1 & 0.1 & 0.1 & 0.1 & 0.05 & 0.72 & 0.62 & 0.1 & 0.480589 & 0.072495 & 0.275054 & 0.321378 \\
\hline 0.1 & 0.1 & 0.1 & 0.5 & 0.05 & 0.72 & 0.62 & 0.1 & 0.442361 & 0.177416 & 0.645167 & 0.328121 \\
\hline 0.1 & 0.1 & 0.1 & 1.0 & 0.05 & 0.72 & 0.62 & 0.1 & 0.428684 & 0.218151 & 0.781848 & 0.330389 \\
\hline 0.1 & 0.1 & 0.1 & 0.1 & 0.01 & 0.72 & 0.62 & 0.1 & 0.487708 & 0.077149 & 0.228508 & 0.319924 \\
\hline 0.1 & 0.1 & 0.1 & 0.1 & 0.03 & 0.72 & 0.62 & 0.1 & 0.484739 & 0.075180 & 0.248199 & 0.320545 \\
\hline 0.1 & 0.1 & 0.1 & 0.1 & 0.05 & 0.72 & 0.62 & 0.1 & 0.480589 & 0.072495 & 0.275054 & 0.321378 \\
\hline 0.1 & 0.1 & 0.1 & 0.1 & 0.05 & 0.72 & 0.62 & 0.1 & 0.480589 & 0.072495 & 0.275054 & 0.321378 \\
\hline 0.1 & 0.1 & 0.1 & 0.1 & 0.05 & 1.0 & 0.62 & 0.1 & 0.491311 & 0.078932 & 0.210680 & 0.319005 \\
\hline 0.1 & 0.1 & 0.1 & 0.1 & 0.05 & 3.0 & 0.62 & 0.1 & 0.505733 & 0.089619 & 0.103808 & 0.316486 \\
\hline 0.1 & 0.1 & 0.1 & 0.1 & 0.05 & 0.72 & 0.24 & 0.1 & 0.451388 & 0.073547 & 0.264530 & 0.187505 \\
\hline 0.1 & 0.1 & 0.1 & 0.1 & 0.05 & 0.72 & 0.62 & 0.1 & 0.480589 & 0.072495 & 0.275054 & 0.321378 \\
\hline 0.1 & 0.1 & 0.1 & 0.1 & 0.05 & 0.72 & 0.78 & 0.1 & 0.488899 & 0.072166 & 0.278343 & 0.372233 \\
\hline 0.1 & 0.1 & 0.1 & 0.1 & 0.05 & 0.72 & 0.62 & 0.1 & 0.480589 & 0.072495 & 0.275054 & 0.321378 \\
\hline 0.1 & 0.1 & 0.1 & 0.1 & 0.05 & 0.72 & 0.62 & 0.5 & 0.759608 & 0.067168 & 0.328319 & 0.283825 \\
\hline 0.1 & 0.1 & 0.1 & 0.1 & 0.05 & 0.72 & 0.62 & 1.0 & 1.022340 & 0.062071 & 0.379290 & 0.254589 \\
\hline
\end{tabular}

The graphical behaviors of Schmidt number $(S c)$ as illustrated in Figure 8-9, reveals that an increase in $S c$ which happen as a result of low molecular diffusivity leads to a reduction in velocity distribution of the fluid and rapidly fall its concentration within the boundary layer. This in turns

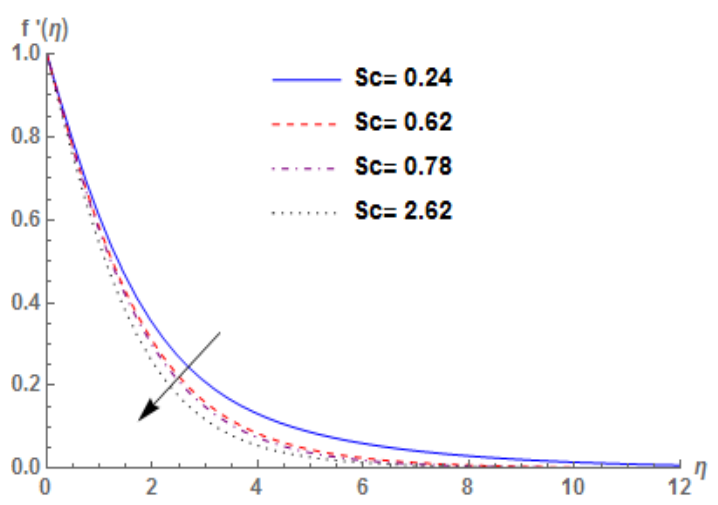

Figure 8. Velocity profiles for different values of $S c$

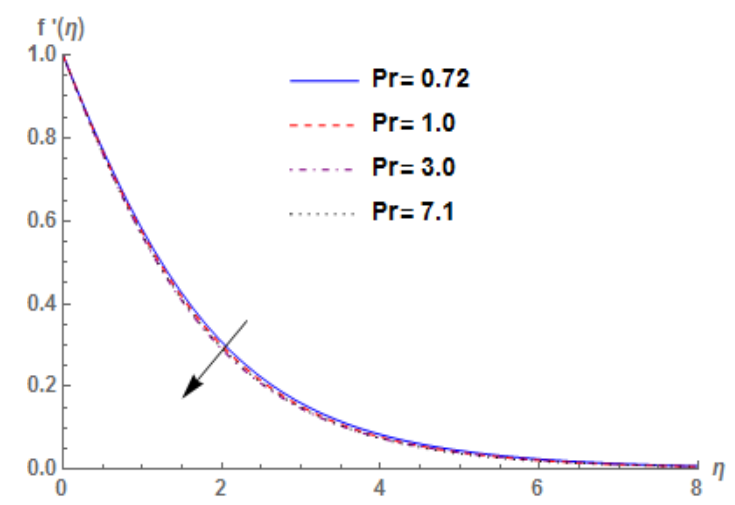

Figure 10. Velocity profiles for different values of $P r$ reduces the diffusion property of the fluid and the concentration boundary layer becomes thinner than the velocity boundary layer thickness. It is observed from Table 2 that the rate of mass transfer improves for higher values of $S c$ as Sherwood number increases.

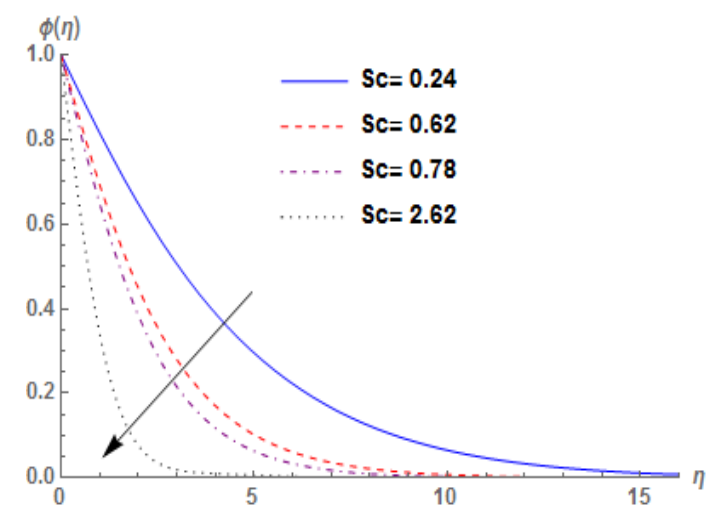

Figure 9. Concentration profiles for different values of $S c$

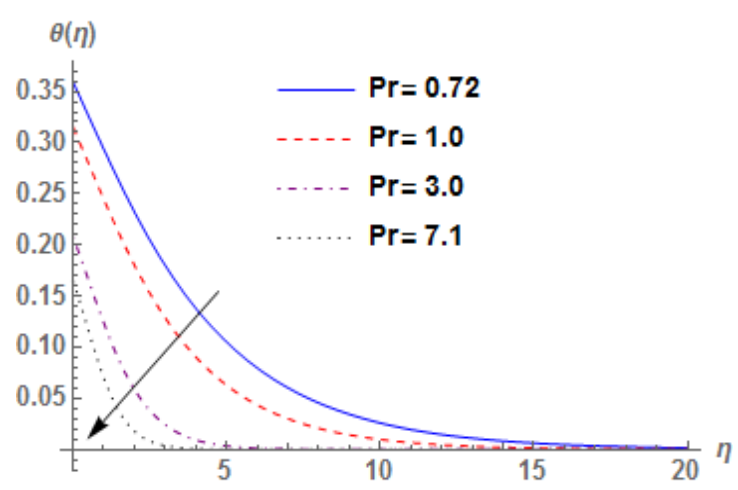

Figure 11. Temperature profiles for different values of $\mathrm{Pr}$ 
The Prandtl number $P r$ which signifies the ratio of momentum diffusivity to thermal diffusivity is presented in Figure 10-11. A rise in $\operatorname{Pr}$ slightly decreases the velocity distribution and rapidly fall its temperature within the boundary layer. This consequently lower the average temperature and thermal boundary layer thickness experiences a reduction. It is noteworthy that the smaller value of $\operatorname{Pr}$ equivalent to increase in the thermal conductivity which causes the heat to diffuse away quickly from the heated surface than the higher value. Therefore, the smaller the Prandtl number, the thicker its thermal boundary layer which leads to reduction in heat transfer rate.

Figure 12-17 depict the effect of buoyancy force $(G r, G c)$ on velocity, temperature and concentration profiles. The

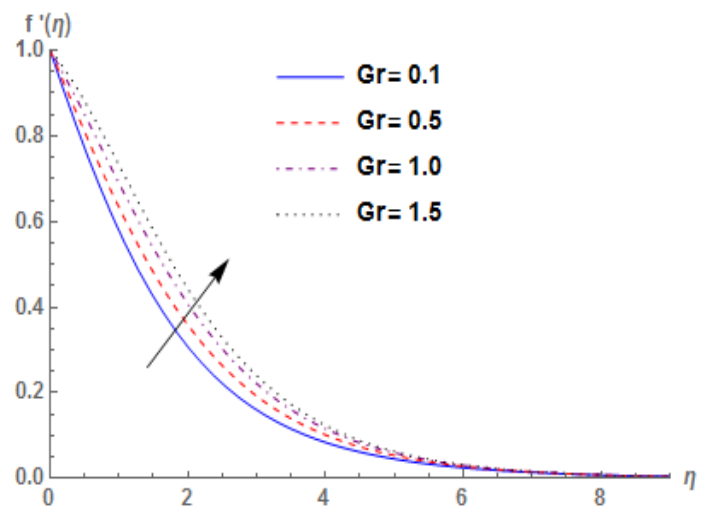

Figure 12. Velocity profiles for different values of $G r$

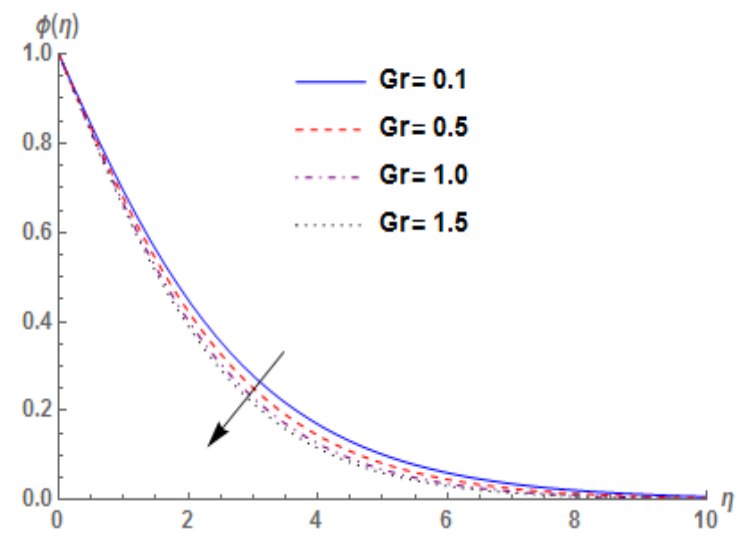

Figure 14. Concentration profiles for different values of $G r$

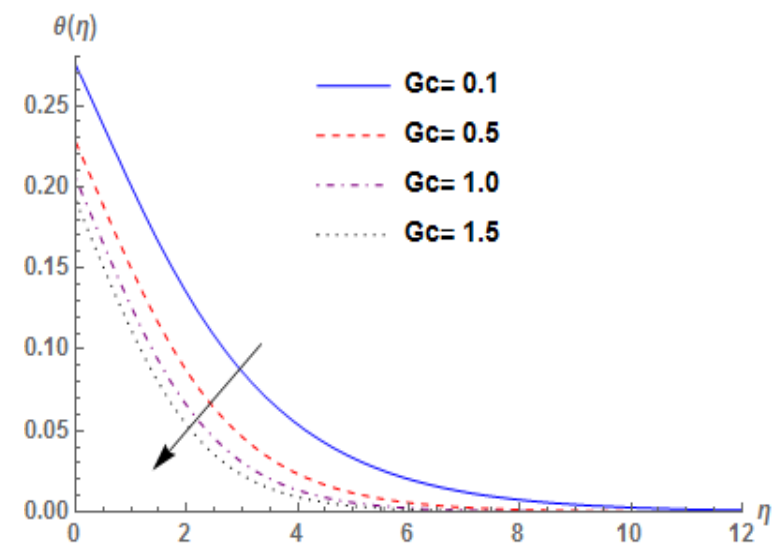

Figure 16. Temperature profiles for different values of $G c$ momentum boundary layer thickness increases due to the enhancement in fluid velocity as a result of the buoyancy force parameters while thermal and concentration boundary layer thickness decrease, owning to the decrease in temperature and concentration of the fluid. It is what mentioning, that the local skin friction and plate surface temperature decrease while the Nusselt and Sherwood numbers increase with the increase in $(G r, G c)$ as shown in Table 2 .

The positive values of thermal Grashof number and Solutal Grashof number correspond to the greater cooling of the surface and shows that the concentration at the plate surface is higher than the free stream concentration respectively.

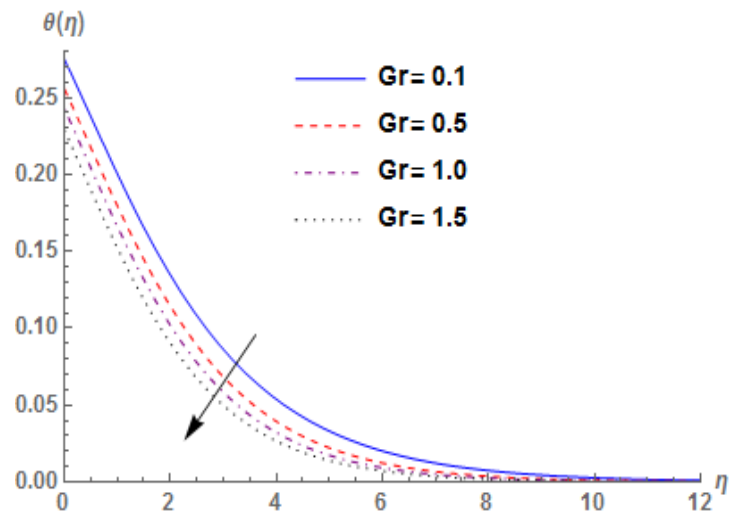

Figure 13. Temperature profiles for different values of $G r$

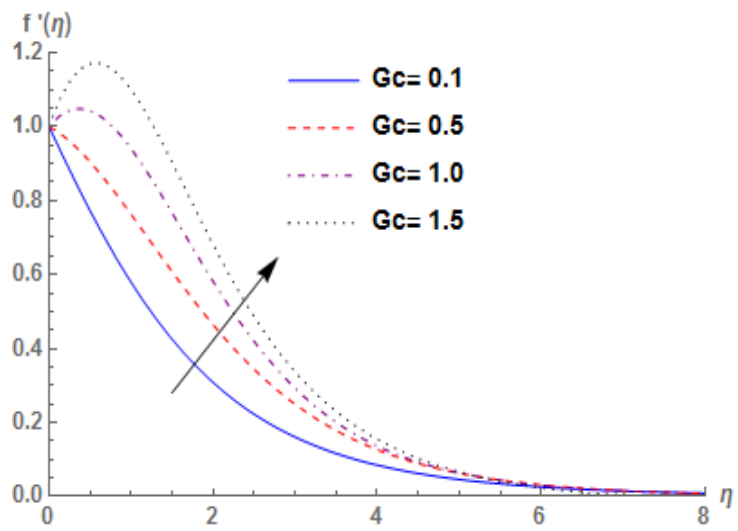

Figure 15. Velocity profiles for different values of $G c$

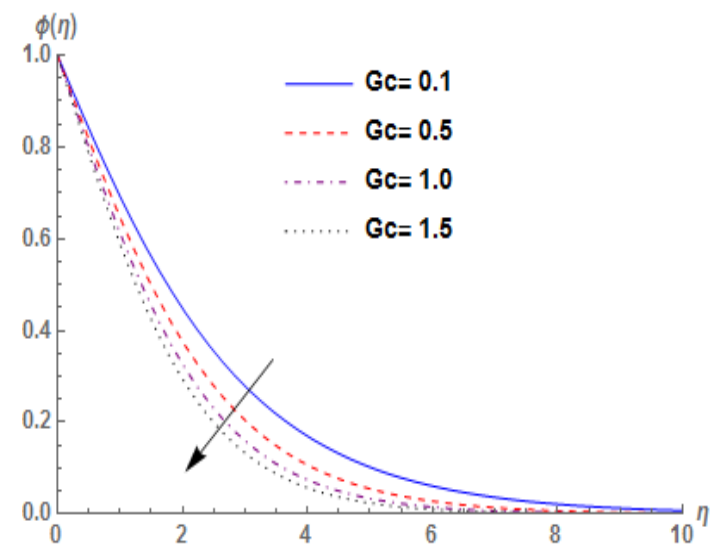

Figure 17. Concentration profiles for different values of $G c$ 
The effect of permeability parameter $\left(P_{s}\right)$ on velocity, temperature and concentration profiles are shown in Figure 18-20 respectively. As expected, increase in $P_{S}$ increase the resistance flow, which in turns causes a reduction in velocity distribution within the boundary layer with a reverse phenomenon in fluid temperature as well as its concentration. However, the magnitude of the local skin-friction and plate surface temperature increase with the increase in $P_{S}$ while the decrease effect is observed in Nusselt and Sherwood numbers (see Table 2). Subject to the increasing effect of $P_{s}$, the thermal boundary layer thickness increases as well as its concentration boundary layer.

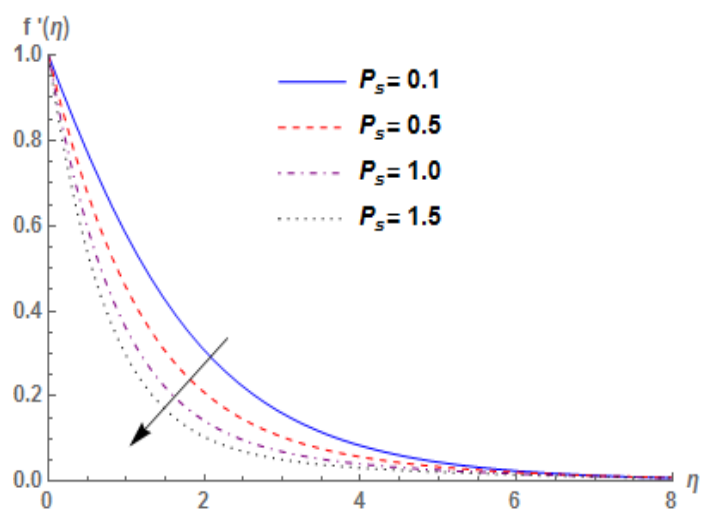

Figure 18. Velocity profiles for different values of $P_{S}$

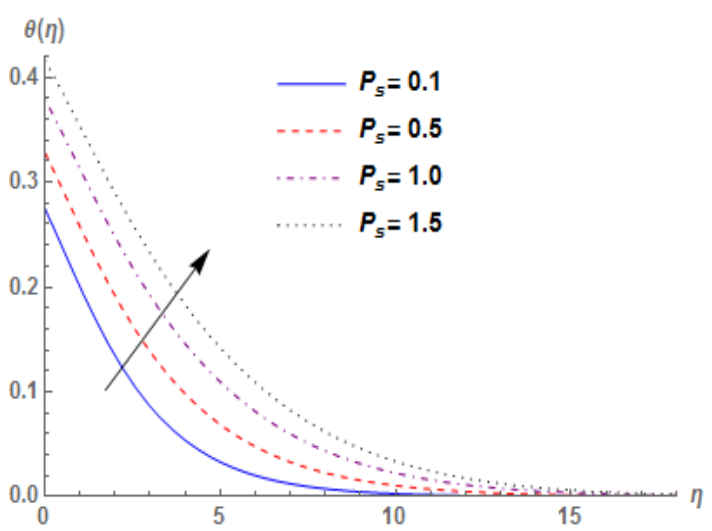

Figure 19. Temperature profiles for different values of $P_{S}$

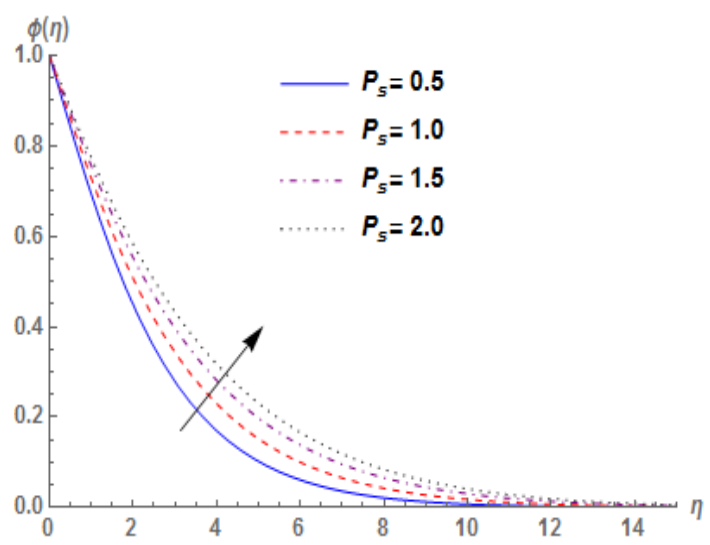

Figure 20. Concentration profiles for different values of $P_{S}$

The presence of heat source $(\delta)$ is to enhance the temperature of the fluid. An increase in internal heat source $\delta$ overshoot the temperature of the fluid at $\delta=0.1$ to its peak value at the plate surface and decreases to a free stream zero value far away from the plate in agreement with the boundary conditions which consequently strengthen the thermal boundary layer thickness. However, the interaction of $\delta$ enhances the molecules of the fluid as the plate surface temperature increases (see Table 2).

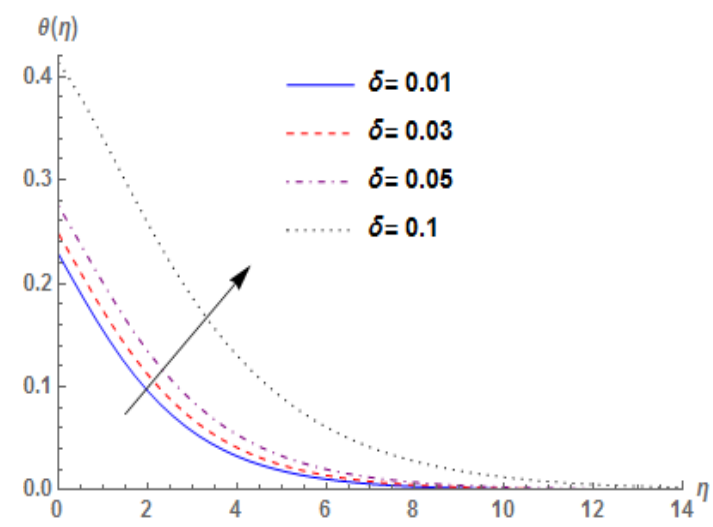

Figure 21. Temperature profiles for different values of $\delta$

The presence of heat source $(\delta)$ is to enhance the temperature of the fluid. An increase in internal heat source $\delta$ overshoot the temperature of the fluid at $\delta=0.1$ to its peak value at the plate surface and decreases to a free stream zero value far away from the plate in agreement with the boundary conditions which consequently strengthen the thermal boundary layer thickness (See Figure 21). However, the interaction of $\delta$ enhances the molecules of the fluid as the plate surface temperature increases (see Table 2).

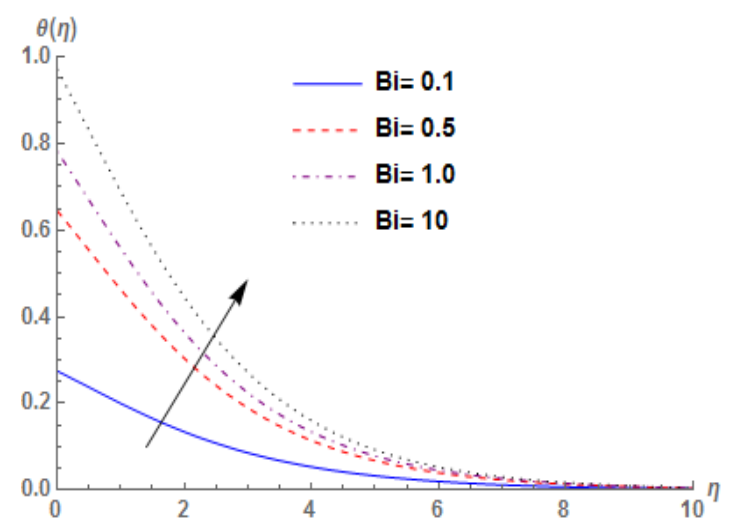

Figure 22. Temperature profiles for different values of $B i$

The interaction of Convective heat transfer parameter as shown in Table 2 improves the Nusselt number greatly which in turns strengthen the rate of heat transfer. However, similar behavior is observed on the plate surface temperature and the fluid temperature (See Figure 22) due to the heat exchange by the hot fluid which in turns strengthen the thermal boundary layer thickness.

\section{CONCLUSION}

A numerical study has been carried out to investigate heat and mass transfer flow past a vertical porous plate in presence of heat source. The partial differential equations which describe the problem were transformed to non-linear ordinary differential equations by Similarity transformation method with the corresponding dimensionless variables. We 
then solve the equations by Homotopy Analysis Method and the results are discussed through graphs and tables for different values of embedding parameters and the following conclusion are drawn among others

(i) Increase in heat source enhance the temperature of the greatly, which shows that the operating temperature is high whenever heat source occurs

(ii) The cooling problem is guaranteed with the positive values of $G r$ which are often encountered in engineering application for the cooling of electronic component and nuclear reactors.

(iii) Increase in convective heat parameter which play an important role in industry and engineering field for drying of materials or components, pioneer great convective heating which in turn magnify the thermal boundary layer thickness.

(iv) smaller value of $\operatorname{Pr}$ equivalent to increase in the thermal conductivity which causes the heat to diffuse away quickly from the heated surface than the higher value.

The major language in Science and Technology discipline is the understanding of the dynamic flow, cooling of the system (cooling of the electronic components) and drying of materials. We then hope that the work will serve as basis for experimental studies.

\section{ACKNOWLEDGEMENT}

The authors acknowledge the thoughtful comments of the anonymous referees for their useful suggestions that led to definite improvement in the paper.

\section{REFERENCES}

[1] Thommaandru, R.R, Kotha, G.B., Hema, S.R., Rao, M.V.S. (2014). Heat source/sink effects of heat and mass transfer of magnetonano fluids over a nonlinear stretching sheet. Advances in Applied Science Research, 5(3): 114-129.

[2] Ibrahim, F.S., Elaiw, A.M., Bakr, A.A. (2008). Effect of the chemical reaction and radiation absorption on unsteady MHD mixed convection flow past a semiinfinite vertical permeable moving plate with heat source and suction. Commun. Nonlinear Sci. Numer. Simul., $13:$ 1056-1066. https://doi.org/10.1016/j.cnsns.2006.09.007

[3] Bakr, A.A. (2011). Effects of chemical reaction on MHD free convection and mass transfer flow of a micropolar fluid with oscillatory plate velocity and constant heat source in a rotating frame of reference. Commun. Nonlinear Sci. Numer. Simul., 16: 698-710. https://doi.org/10.1016/j.cnsns.2010.04.040

[4] Olanrewaju, P.O., Arulogun, O.T., Adebimpe, K. (2012). Internal heat generation effect on thermal boundary layer with a convective surface boundary condition. Aim J Fluid Dynam., 2(1): 1-4. https://doi.org/10.5923/j.ajfd.20120201.01

[5] Crepeau, J.C., Clarksean, R. (1997). Similarity solutions of natural convection with internal heatgeneration. Trans ASME J Heat Transf., 119: 184-185. https://doi.org/10.115/1.2824086

[6] Ashwini, G., Eswara, A.T. (2012). MHD Falkner Skan boundary layer flow with internal heat generation or absorption. World Academy of Science Engineering and Technology, 65: 687-690.

[7] Makinde, O.D. (2011). Similarity solution for natural convection from a moving vertical plate with internal heat generation and a convective boundary condition. Thermal Science, 15(1): https://doi.org/10.2298/TSCI11S1137M

S137-S143.

8] Azim, M.A., Mamun, A.A., Rahman, M.M. (2010). Viscous Joule heating MHD-conjugate heat transfer for a vertical flat plate in the presence of heat generation. International Communications in Heat and Mass Transfer, 37(6): 666-674. https://doi.org/10.1016/j.icheatmasstransfer.2010.02.00 2

[9] Rashid, A., Waqar, A.K. (2013). Effect of Viscous Dissipation and internal heat generation/absorption on heat transfer flow over a moving wedge with convective boundary condition. Heat Transfer-Asian Research, 42(7): 589-602. https://doi.org/10.1002/htj.21055

[10] Machireddy, G.R., Polarapu, P., Bandari, S. (2015). Effects of viscous dissipation and heat source on unsteady MHD flow over a stretching sheet. Ain Shams Engineering Journal, 4: 2090-4479. https://doi.org/10.1016/j.asej.2015.04.006

[11] Rana, R., Bhargav, R. (2011). Numerical study of heat transfer enhancement in mixed convection flow along a vertical plate with heat source/sink utilizing nanofluids. Commun. Nonlinear Sci. Numer. Simulat., 16: 43184334. https://doi.org/10.1016/j.cnsns.2011.03.014

[12] Kasmani, R.M., Sivasankaran, S., Bhuvaneswari, M., Siri, Z. (2016). Effect of chemical reaction on convective heat transfer of boundary layer flow in nanofluid over a wedge with heat generation/absorption and suction. Journal of Applied Fluid Mechanics, 9(1): 379-388.

https://doi.org/18869/acadpub.jafm.68.224.24151

[13] Mohamad, R.A (2009). Double diffusive convection radiation interaction on unsteady MHD flow over a vertical moving porous plate with heat generation and Soret effect. Applied Mathematical Sciences, 13: 629651.

[14] Patil, P.M., Kulkarni, P.S. (2008). Effects of chemical reaction on free convective flow of a polar fluid through a porous medium in the presence of internal heat generation. International Journal of Thermal Science, 47(8): 1043-1054. https://doi.org/10.1016/j.ijthermalsci.2007.07.013

[15] Ramana, R.G.V., Murthy, C.V.R., Reddy, N.B. (2010). Mass transfer and radiation effects of unsteady MHD free convective fluid flow embedded in porous medium with heat generation/absorption. Journal of Applied Mathematics and Fluid Mechanics, 2(1): 85-98.

[16] Sharma, P.R., Singh, G., (2008). Unsteady MHD free convective flow and heat transfer along a vertical porous plate with variable suction and internal heat generation. International Journal of Advances in Applied Mathmatics and Mechanics, 4(5): 1-8.

[17] Mamta, T., Krishna, M.V. (2014). Thermal radiation effect on an unsteady MHD free convective chemically reacting viscous dissipative fluid flow past an infinite vertical moving porous plate with Heat source. IOSR Journal of Mathematics, 10(3): 89-105. https://doi.org/10.9790/5728-103189105

[18] Sharma, P.R., Sharma, K., Mehta, T. (2014). Radiative and free convective effects on MHD flow through a 
porous medium with periodic wall temperature and heat generation or absorption. Int. J. of Mathe. Archive, 5(9): 119-128.

[19] Liao, S.J. (2003). Beyond perturbation: An introduction to Homotopy Analysis Method. Boca Raton, Fla., USA: Chapman and Hall.

[20] Liao, S.J. (2003). On the analytic solution of magnetohydrodynamic flows of non-Newtonian fluids over a stretching sheet. J Fluid Mech., 488: 189-212. https://doi.org/10.1017/S0022112003004865

[21] Olubode, K.K, Tosin, O, Adeola, J.O, Isaac, L.A. (2016). Homotopy analysis of MHD free convective micropolar fluid flow along a vertical surface embedded in Non-Darcian thermally-stratified medium. Open Journal of Fluid Dynamic, 6: 198-221. https://doi.org/10.4236/ojfd.2016.63016

[22] Adhikari, A., Maiti, A.K. (2014). MHD micropolar fluid flow towards a vertical surface in a presence of heat source/sink under radiative heat flux. Journal of the International Mathematical Virtual Institute, 4: 1-25. https://doi.org/11.7251/JIMVI140101A

[23] Makinde, O.D. (2010). On MHD heat and mass transfer over a moving vertical plate with a corrective surface boundary condition. Can. J. Chem. Eng., 88: 983-990. https://doi.org/10.1002/cjce.20369

\section{NOMENCLATURE}

Ha Magnetic field parameter

Gr Local thermal Grashof number

Gc Local solutal Grashof number

$B i \quad$ Local convective heat transfer parameter

Pr Prandtl number

$\delta \quad$ Heat source parameter

$P_{S} \quad$ porosity parameter

$Q_{0}$ volumemetric heat generation/ absorption rate

Sc Schmidt number

\section{Greek symbols}

$\eta$ Similarity variable

$\psi$ Stream function 